Claremont Colleges

Scholarship@ Claremont

All HMC Faculty Publications and Research

HMC Faculty Scholarship

$2-1-2001$

\title{
Particle Size Determination: An undergraduate lab in Mie scattering
}

I. Weiner' 01

Harvey Mudd College

M. Rust '01

Harvey Mudd College

Thomas D. Donnelly

Harvey Mudd College

\section{Recommended Citation}

I. Weiner, M. Rust and T.D. Donnelly, "Particle size determination: An undergraduate lab in Mie scattering," Am. J. Phys 69, 129 (2001). doi: 10.1119/1.1311785

This Article is brought to you for free and open access by the HMC Faculty Scholarship at Scholarship @ Claremont. It has been accepted for inclusion in All HMC Faculty Publications and Research by an authorized administrator of Scholarship @ Claremont. For more information, please contact scholarship@cuc.claremont.edu. 


\section{AAPT AMERICAN \\ JOURNAL}

\section{Particle size determination: An undergraduate lab in Mie scattering}

I. Weiner, M. Rust, and T. D. Donnelly

Citation: Am. J. Phys. 69, 129 (2001); doi: 10.1119/1.1311785

View online: http://dx.doi.org/10.1119/1.1311785

View Table of Contents: http://ajp.aapt.org/resource/1/AJPIAS/v69/i2

Published by the American Association of Physics Teachers

\section{Related Articles}

Improving the quantification of Brownian motion

Am. J. Phys. 81, 485 (2013)

Photon charge experiment

Am. J. Phys. 81, 436 (2013)

Collimated blue light generation in rubidium vapor

Am. J. Phys. 81, 442 (2013)

The Wiimote on the Playground

Phys. Teach. 51, 272 (2013)

Helicopter Toy and Lift Estimation

Phys. Teach. 51, 310 (2013)

\section{Additional information on Am. J. Phys.}

Journal Homepage: http://ajp.aapt.org/

Journal Information: http://ajp.aapt.org/about/about_the_journal

Top downloads: http://ajp.aapt.org/most_downloaded

Information for Authors: http://ajp.dickinson.edu/Contributors/contGenlnfo.html

\section{ADVERTISEMENT}

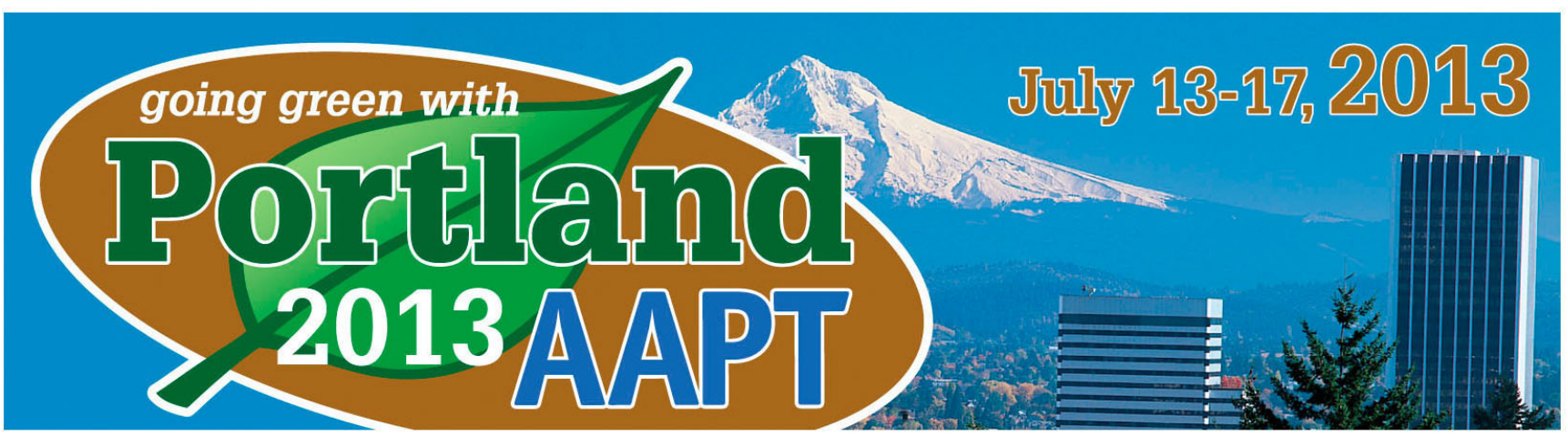




\title{
Particle size determination: An undergraduate lab in Mie scattering
}

\author{
I. Weiner, M. Rust, and T. D. Donnelly \\ Harvey Mudd College, Department of Physics, Claremont, California 91711
}

(Received 22 March 2000; accepted 1 June 2000)

\begin{abstract}
A technique for determining the size of microscopic spherical particles using light scattering is presented as an undergraduate physics lab. Scatterer size is determined from angular scattering distribution measurements of laser light scattered from a dilute suspension of latex spheres with diameters of $4.99 \pm 0.05$ and $6.038 \pm 0.045 \mu \mathrm{m}$. Previous experiments of this type used approximate theoretical corrections and required the construction of specialized sample cells to minimize complicating effects. As a significant improvement to these, we generate angular scattering distributions from Mie theory and, using an accurate numerical procedure, correct these distributions for Snell's law and foreshortening effects. Scatterer size is then determined using a fast, robust fitting algorithm to compare these corrected angular scattering distributions to measured angular scattering distributions. We fit the scatter from a solution of $6.04-\mu \mathrm{m}$-diam spheres to spheres of $5.95 \pm 0.11 \mu \mathrm{m}$ diameter, and that from a solution of $4.99-\mu \mathrm{m}$ spheres to 4.85 $\pm 0.15 \mu \mathrm{m}$. Additionally, scattering data for a 2:1 mixture of spheres of diameters 4.99 and $6.04 \mu \mathrm{m}$ are taken, and after numerical adjustment for Snell's law and foreshortening effects, good agreement with theory is obtained. (C) 2001 American Association of Physics Teachers.
\end{abstract}

[DOI: 10.1119/1.1311785]

\section{INTRODUCTION}

It is straightforward to calculate the cross section for plane wave light scattering from a uniform dielectric sphere using classical electromagnetic theory. One possible application of these results is the determination of particle sizes by measuring the intensity of angular scattering distributions. The ability to measure the size of particles with diameters on the order of microns with such elementary measurement techniques is indeed impressive.

In this paper we propose a simple yet accurate and versatile method for the determination of spherical particle sizes on the order of $1 \mu \mathrm{m}$ diameter that may be implemented easily in an undergraduate physics lab. In the past decade, a number of particle sizing techniques have been developed that use a variety of approaches. Sizing may be quite accurately carried out using electron microscopy, for example, but this is prohibitively expensive for use in an undergraduate lab. A number of less expensive techniques involving laser-light scattering have been developed using Mie scattering theory and other methods. Dovichi and Zarrin, for example, have accurately sized submicron particles being carried at constant velocities using laser Doppler velocimetry. ${ }^{1}$ However, it is cumbersome to maintain particles at a constant velocity. Wang and Hallett, and others, have successfully determined particle size distributions through an inversion of the extinction spectrum of a static solution of particles. ${ }^{2}$ Unfortunately, an undergraduate physics lab is unlikely to have a convenient method of obtaining extinction data for a large range of frequencies, which is, of course, an integral part of the inversion technique. For these reasons, the techniques discussed above are inappropriate for our purposes.

With the rapid increase in computer processor power in the past few years, computational methods for fitting a single sphere size or distribution of sizes from measured angular scattering distributions have been developed. These methods use fairly advanced Monte Carlo or genetic algorithm methods. ${ }^{3}$ We present here a convenient technique for measuring angular scattering distributions for a static suspension of micron-size spheres using only standard lab equipment. We also present a computational technique for fitting angular scattering distributions that is substantially simpler than those mentioned above, and is more applicable to the task at hand. Because the spheres are held in an aqueous suspension inside a cuvette, we have also developed numerical techniques for correcting the theoretical scattering curves for Snell's law refraction and for the "foreshortening'" effect produced when scattered light from a horizontal column of scatterers is viewed from different angles. In the past, a similar experiment was presented as a potential undergraduate physics laboratory by Drake and Gordon ${ }^{4}$ using approximations to the Mie theory and approximate analytical corrections for Snell's law. To avoid the foreshortening effect and maximize the accuracy of their approximate Snell's law correction, it was necessary to place the spheres in a specially constructed sample cell of unusually small thickness. These approximations met with only limited success; there were substantial deviations from theory, mostly due to the approximations of the Mie theory. In addition, no means of fitting the data was proposed. It is now possible, due to the speed of modern computer processors, to employ the routines discussed in this paper to actively fit data to arbitrarily accurate theoretical curves within a matter of seconds, allowing real time fitting during the data collection process. In addition, no special sample cell is required to contain the solution of scatterers; an ordinary cuvette of square cross section suffices.

\section{THEORY}

In a solution of scattering spheres, if the concentration of spheres is not too high, we may approximate their collective scattering as resulting from a large number of independent scatterers, and as such, the scattering curve should simply be some multiple of the curve for a single sphere. A concentration of such spheres is said to be in the "single scattering regime." The theoretical expression for the intensity of light scattered from a spherical particle to a given angle, the angular scattering distribution, is given by the Mie theory, ${ }^{5,6}$ 


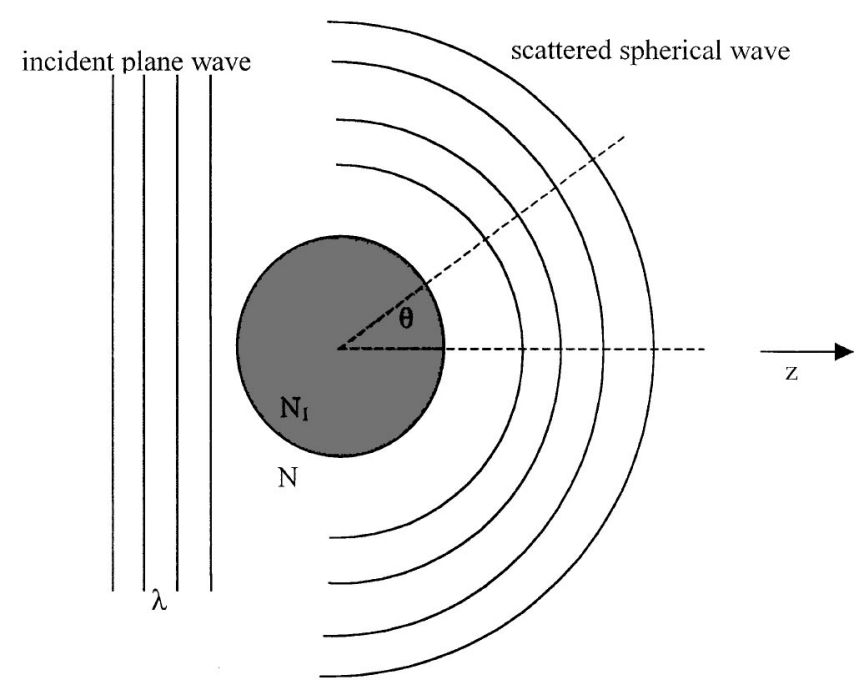

Fig. 1. Illustration of Mie scattering. $N$ and $N_{1}$ refer to the indices of refraction of the medium surrounding the scatterer and the particle material, respectively; $\lambda$ is the wavelength of the incident light.

and is well known. The solution is obtained by solving the problem of a plane electromagnetic wave incident on a uniform uncharged sphere of arbitrary radius and refractive index, as shown schematically in Fig. 1. The basic idea is to expand the incident plane wave in a Fourier series using appropriate basis functions that satisfy Maxwell's equations in spherical coordinates; spherical coordinates are used due to the geometry of the scatterers. We then apply suitable boundary conditions at the surface of the sphere in order to deduce the Fourier expansions of the scattered fields inside and outside the sphere. Because the derivation of the scattering coefficients is quite long, we defer it to Appendix A. (This derivation is mathematically advanced and will be a challenge for all but the most advanced undergraduateshowever, it is our experience that undergraduates can master this material. We include the derivation, in its entirety, so that the precocious student may find the relevant theoretical framework and laboratory discussion self-contained in a single document.) As shown there, the final solution for the field outside the sphere is given by

$$
\begin{aligned}
& E_{s}=\sum_{n=1}^{\infty} E_{n}\left(i a_{n} \mathbf{N}_{e 1 n}^{(3)}-b_{n} \mathbf{M}_{o 1 n}^{(3)}\right), \\
& \mathbf{H}_{s}=\frac{k}{\omega \mu} \sum_{n=1}^{\infty} E_{n}\left(i b_{n} \mathbf{N}_{o 1 n}^{(3)}+a_{n} \mathbf{M}_{e 1 n}^{(3)}\right),
\end{aligned}
$$

where the coefficients are given by

$$
\begin{aligned}
a_{n} & =\frac{\mu m^{2} j_{n}(m x)\left[x j_{n}(x)\right]^{\prime}-\mu_{1} j_{n}(x)\left[m x j_{n}(m x)\right]^{\prime}}{\mu m^{2} j_{n}(m x)\left[x h_{n}^{(1)}(x)\right]^{\prime}-\mu_{1} h_{n}^{(1)}(x)\left[m x j_{n}(m x)\right]^{\prime}}, \\
b_{n} & =\frac{\mu_{1} j_{n}(m x)\left[x j_{n}(x)\right]^{\prime}-\mu j_{n}(x)\left[m x j_{n}(m x)\right]^{\prime}}{\mu_{1} j_{n}(m x)\left[x h_{n}^{(1)}(x)\right]^{\prime}-\mu h_{n}^{(1)}(x)\left[m x j_{n}(m x)\right]^{\prime}} .
\end{aligned}
$$

Here $j_{n}$ 's are the spherical Bessel functions of the first kind, $h_{n}$ 's are the spherical Hankel functions, $\mu_{1}$ and $\mu$ are the magnetic permeability of the sphere and surrounding medium, respectively, $\mathbf{N}$ and $\mathbf{M}$ are the vector spherical harmonics whose expressions in terms of commonly known basis functions are given in Appendix $\mathrm{A}$, and the prime indicates a derivative. If we take $\mu_{1}=\mu$, both drop out of the

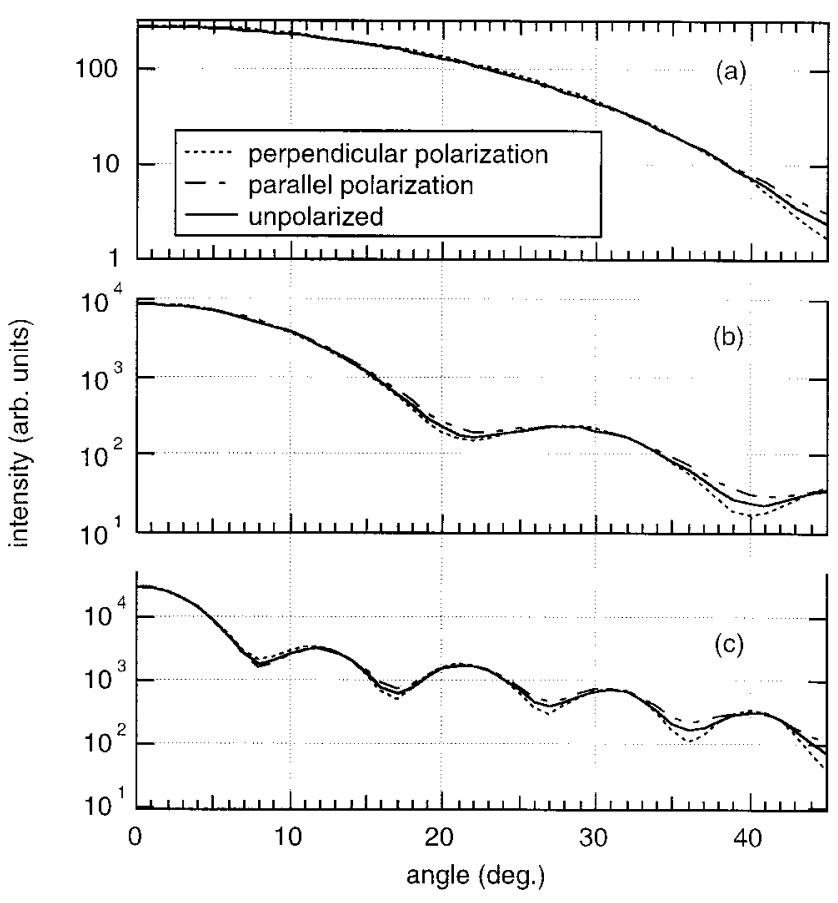

Fig. 2. The angular scattering distribution for incident light which is unpolarized, parallel, and perpendicularly polarized. Scattered light is shown for particles of size parameter $x=$ (a) 5, (b) 10, and (c) 20 .

expressions. The coefficients of Eq. (2) are dependent upon the quantity

$$
x=k a=\frac{2 \pi N a}{\lambda},
$$

where $a$ is the radius of the sphere, $\lambda$ is the wavelength of incident light in vacuum, and $N$ is the refractive index of the surrounding medium. The quantity $x$, called the "size parameter," is what determines the scattering curve, rather than the radius or wavelength independently. It is this parameter to which we fit in our routines. We display sample plots of the theoretical angular scattering distribution for a range of size parameters in Fig. 2. The size parameters $x=5,10$, and 20 correspond, with our setup ( $\lambda=633 \mathrm{~nm})$, to sphere diameters of $0.76,1.51$, and $3.03 \mu \mathrm{m}$, respectively. Although the intensities are expressed in arbitrary units, the scaling of intensities is the same for the three plots. Thus, we can note that as the scatterer's size parameter increases, scatter in the forward direction increases dramatically. Also, we begin to see oscillatory behavior in the angular scattering distribution only at size parameters around ten or higher; thus spheres of size parameter smaller than this are more difficult to fit accurately.

\section{EXPERIMENTAL METHODS}

Figure 3 shows a diagram of the experimental setup. An unpolarized 1-mW helium-neon $(\mathrm{He}-\mathrm{Ne})$ laser (Edmund Scientific, \#F61337), with a wavelength of $632.8 \mathrm{~nm}$, is directed through the center of a glass cuvette with square cross section (a square of side length $1 \mathrm{~cm}$ ), containing the sphere suspension (one drop of spheres in $100 \mathrm{ml}$ of water; $\sim 10^{10}$ spheres $/ \mathrm{ml}$ ). The suspension is created using filtered water to eliminate any scatterers other than the latex spheres. We directed the beam using two mirrors, which aided in laser 


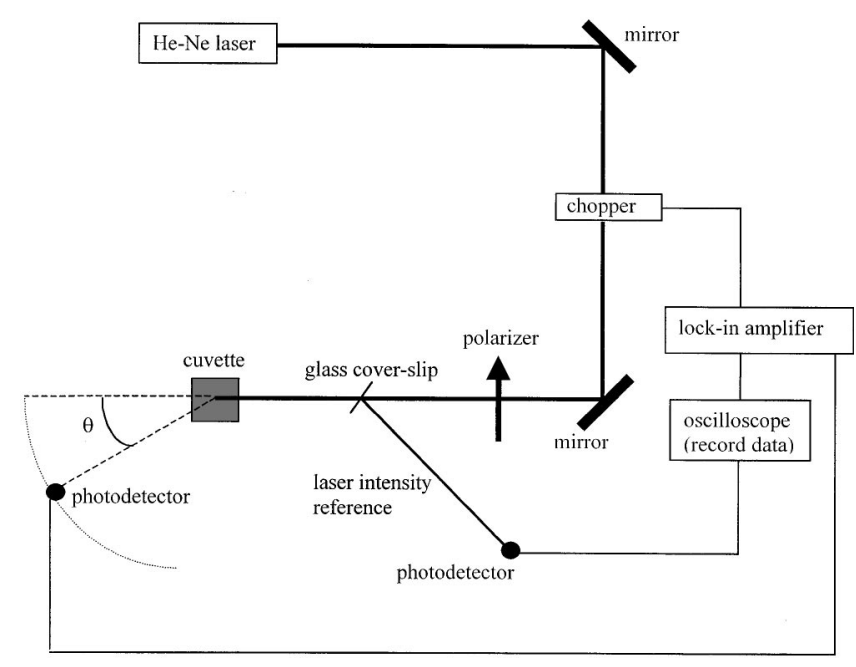

Fig. 3. Configuration of the experiment. The lock-in amplifier is helpful in increasing the signal-to-noise ratio of the scattered light intensity, but is not essential. Both the scattered light (signal output from the lock-in amplifier) and the reference intensity (signal from the photodetector looking at the glass cover-slip) are recorded as a function of emission angle $\theta$.

alignment and reduced the length of breadboard required for the experiment. The cuvette was mounted directly above a rotation stage using optical posts and other common equipment. A photodetector (ThorLabs, \#PDA55) was attached to the rotation stage via a rail. The photodetector was placed $24.5 \mathrm{~cm}$ from the center of the cuvette. Our setup allowed the photodetector to measure scattered light at angles as small as $4^{\circ}$; at angles smaller than this, the attenuated laser beam was incident upon the photodetector. We were able to achieve an angular resolution of $\pm 1^{\circ}$. This resolution proved adequate for our purposes; however, it would be a trivial matter to increase it further by closing the iris at the front of the photodetector. Data were collected for angles ranging from $4^{\circ}$ to between $24^{\circ}$ and $40^{\circ}$.

The latex spheres are an essentially monodispersed sample of a NIST certified size (Duke Scientific Co., \#4206A: $6.038 \pm 0.045 \mu \mathrm{m}$ spheres, \#4205A: $4.99 \pm 0.05 \mu \mathrm{m}$ spheres), and have an index of refraction of 1.59 at $589 \mathrm{~nm}$. We found it important to clean the cuvette sides with methanol before taking measurements, in order to reduce flare from dirt, fingerprints, etc. In order to correct for these potential problems, we took a background data set (measured scattering from pure water) before our data-taking runs. The intensity of the background was found to be negligible compared to the scattered signal.

It is important that the concentration of the spheres is not so high that multiple scattering is significant, so that we may treat the suspension as a large number of independent, noninteracting Mie scatterers. We took multiple data sets a few times at various concentrations to ensure the curves differed only by a multiplicative constant as expected. We also checked for multiple scattering by eye, as a "halo" of scatter around the beam is visible when significant multiple scattering takes place.

In order to increase the signal-to-noise ratio of the data and to avoid a background signal from room lights, we used a chopper and lock-in amplifier (SRS, \#SR830 DSP). However, if such equipment is not available, a He-Ne optical filter (Edmund Industrial Optics, \#J43-081) affixed to the photodetector will provide adequate screening of room lights, and can save considerable expense.

We found that the $\mathrm{He}-\mathrm{Ne}$ laser intensity sometimes fluctuated substantially in the relative contributions from parallel and perpendicular polarizations, and in absolute intensity if adequate warm up time was not allowed. Because scattering intensities are polarization dependent, we found it useful to run the laser through a polarizer (Edmund Industrial Optics, \#J52-574) and then through a slightly angled glass cover-slip that directed a small fraction of the beam to a reference photodetector. Thus we were able to correct for fluctuations in the intensity of the particular polarization by normalizing to the reference intensity.

\section{NUMERICAL METHODS}

The analysis of experimental data was heavily dependent upon numerical methods, since the expressions for the theoretical Mie scattering curves are complicated [see Eqs. (1) and (2)]. In addition, we needed to correct for Snell's law refraction and foreshortening, as discussed below, using numerical methods, as we know of no exact (or even reliably accurate) analytic correction factor for these effects. As mentioned above, Drake and Gordon employed an approximate analytical correction for Snell's law that required a specialized sample cell to contain the spheres in order to maximize the accuracy of the approximation and to avoid the foreshortening problem. Fortunately, these problems were resolved with little difficulty and great accuracy using the numerical techniques we have developed, thus allowing us to employ a simple square cuvette instead of a specially constructed sample cell. We rely, then, on the computer to deconvolve data; while this adds to the computational complexity of the experiment, it obviates the need for collecting/imaging optics and therefore greatly simplifies the experimental setup.

It is extremely difficult, if not impossible, to calculate the absolute theoretical scattering intensity with any real accuracy, because it depends on many factors, such as the density of spheres in the suspension, the quantum efficiency of the photodetector, geometry of the cuvette, and the absolute intensity of the incoming beam. Therefore, we decided, rather, to have our fitting routine fit our data to within an arbitrary scaling factor (in addition to fitting the size parameter).

Before fitting the data, however, we need the ability to generate the appropriate theoretical curves. All initial scattering intensities were calculated using MIEV0, ${ }^{7}$ a program which generates angular scattering distributions for some scaling factor, given a size parameter $x$ (we refer to these as "uncorrected" curves in that Snell's law refraction and foreshortening effects are not accounted for in the MIEV0 code). For ease of use and in order to integrate it into our other programs, which were written in $\mathrm{C}$ and $\mathrm{C}++$, we used the public domain package F2C to convert the FORTRAN into ANSI C. The program was used to generate tables of scattering intensities over relevant ranges of angles and size parameters in increments of $1^{\circ}$ and $0.25^{\circ}$, respectively.

After generating the theoretical scattering curves, we needed to correct for refraction and foreshortening effects before we could compare them to the data. As it leaves the water of the cuvette, the scattered light refracts according to Snell's law, and, in addition, there is a "foreshortening" effect which causes the perceived scattering angle of various scatterers seen by the photodetector to vary with detection 


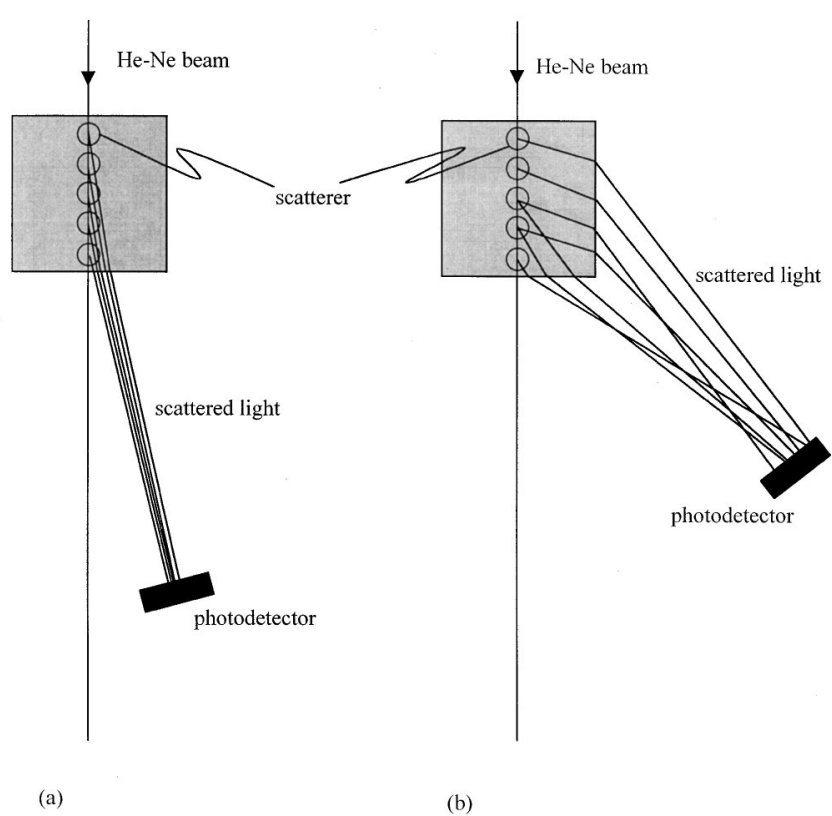

Fig. 4. A diagram of Snell's law refraction and foreshortening effects. (a) At small detection angles, the rays from various scatterers that make it to the photodetector are roughly all emitted at the same angle. (b) At larger detection angles, however, the scattering angle varies from scatterer to scatterer. In addition, the scattered light from some regions can hit the photodetector from two different scattering angles.

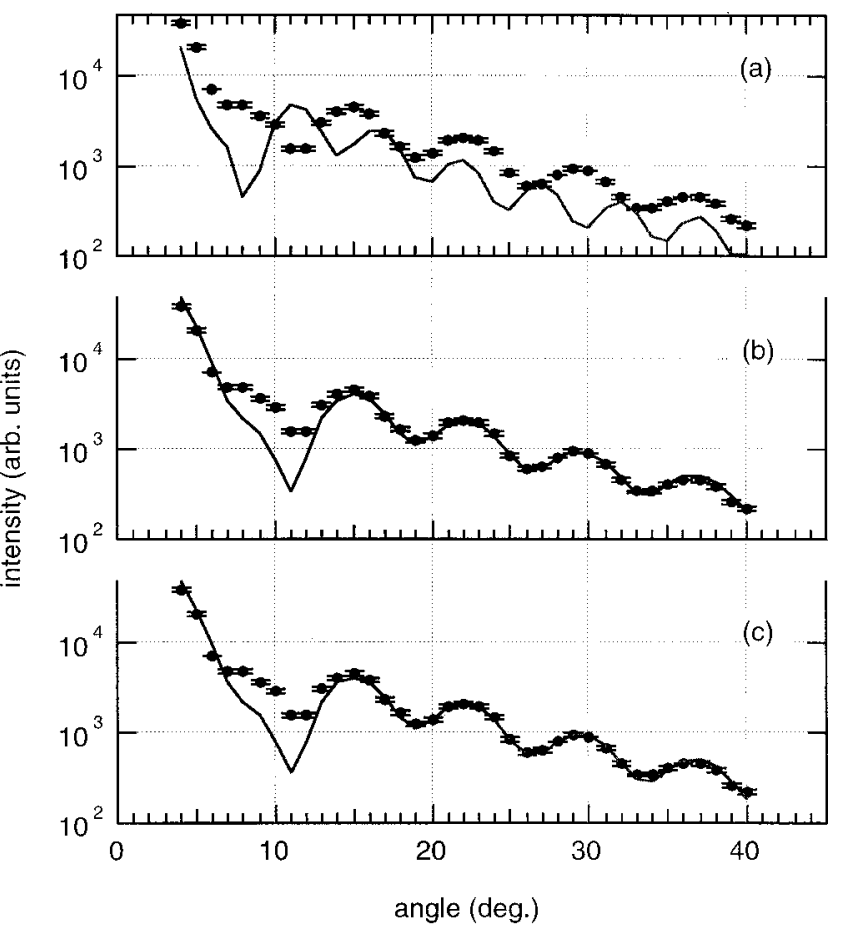

Fig. 5. Correcting Mie theory fits for refraction at cuvette face and foreshortening effects. Data are taken with 6.04- $\mu$ m-diam latex spheres $(x$ $=39.9)$ using unpolarized $\mathrm{He}-\mathrm{Ne}$ light $(\lambda=633 \mathrm{~nm})$. The best fit is 5.95 $\pm 0.11 \mu \mathrm{m}(x=39.25 \pm 0.75)$. (a) Uncorrected; (b) corrected for refraction (Snell's law) at cuvette interface; (c) corrected for refraction at cuvette interface and foreshortening effect. Clearly, for these data, Snell's law refraction dominates the foreshortening effect. angle, as shown in Fig. 4. We wrote a program that simultaneously corrected for both of these problems. Figure 5 shows a data set compared to theory at various stages of the correction. The effect of the Snell's law correction is quite impressive, while the plots show that the foreshortening correction is far less significant for these data, a result which is not obvious. It should be noted that for different experimental geometries (particularly those with a larger cuvette-side to rotation-arm ratio), the foreshortening effect may indeed be substantial. One factor we did not take into account is the variation with angle of the transmission coefficient of glass, since it can easily be shown using the Fresnel coefficient that this effect is negligible for the range of angles we used.

The fitting program we developed is quite simple. It takes as input the corrected theoretical scattering tables and the angular scattering data to be fit. For each size parameter in the theory table, it finds the scaling factor that minimizes $\chi^{2}$ using standard numerical techniques for curve fitting over a single free parameter. It then takes the minimum $\chi^{2}$ over all size parameters to get the final fit. The accuracy of the fit is in principle only limited by the spacing of the size parameters in the theory table constructed.

The programs and source code discussed above are available upon request from the authors [tom_donnelly@hmc.edu]. For a more in-depth discussion of the theory, approach, and numerical methods behind Snell's law and foreshortening correction, see Appendix B.

\section{DATA AND RESULTS}

We took scattering measurements as a function of angle for two sphere diameters, 6.04 and $4.99 \mu \mathrm{m}$, at various polarizations of the incident light. We also took scattering data for a 2:1 mixture of spheres of diameters 4.99 and $6.04 \mu \mathrm{m}$. With the combination of our refraction and foreshortening corrections and our fitting algorithm, we fit the scattering data for the 6.04- and $4.99-\mu \mathrm{m}$ spheres to $5.95 \pm 0.11$ and $4.85 \pm 0.15 \mu \mathrm{m}$, respectively. Results are plotted in Figs. $5-7$.

For both the 4.99- and $6.04-\mu \mathrm{m}$ cases, the theoretical curve dips sharply at a low angle before oscillating, while the measured data drop far less sharply. This is probably a consequence of finite angular resolution, as the width of the drop is roughly $1^{\circ}$, and thus is not properly resolved with our apparatus. However, except in these small angular regions, the theoretical plots show good agreement with the scattering measurements.

If our techniques are used to fit data to an unknown sphere size, it is important to have some judge of how good the fit is-how close is our best fit relative to our second best, for example? For this purpose, the simple approach taken in our fitting routine becomes a great asset, as it allows us to easily calculate and plot $\chi^{2}$ (minimized with respect to the multiplicative parameter) as a function of size parameter $x$. Such a plot is shown in Fig. 8 for the 4.99- and 6.04- $\mu \mathrm{m}$ sphere data. It shows the $\chi^{2}$ "basin" in which the best fit resides. The lowest basin is at size parameter $x=39.25$, which corresponds to our best fit sphere diameter of $5.95 \mu \mathrm{m}$. The width of the basin, roughly \pm 0.75 , determines an uncertainty of the particle diameter of $\pm 0.11 \mu \mathrm{m}$, consistent with the manufacturer's value of $6.04 \mu \mathrm{m}$. The fact that our lowest basin is substantially lower than other basins gives us confidence that the general range of size parameters we calculate with our correction and fitting algorithms is indeed correct. 


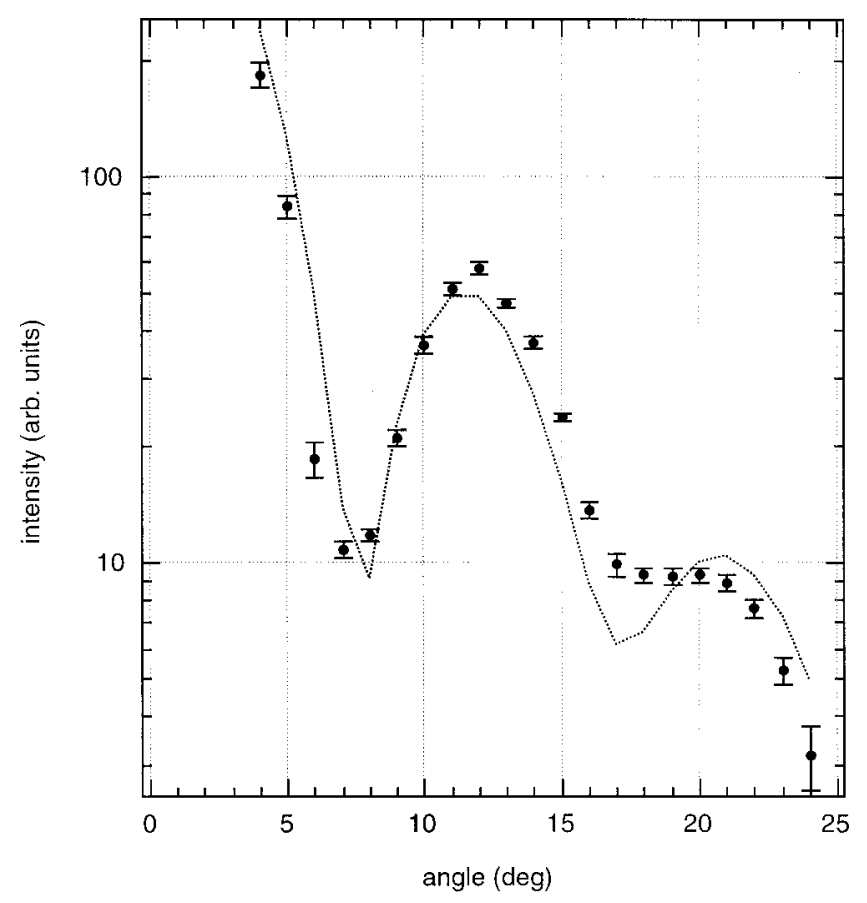

Fig. 6. Scattering data for latex spheres with $4.99 \mu \mathrm{m}$ diameter $(x$ $=32.9)$; incident laser light $(\lambda=633 \mathrm{~nm})$ is perpendicularly polarized. The best fit is to scatterers with $4.85 \pm 0.15 \mu \mathrm{m}$ diameter $(x=32.00 \pm 1.00)$.

The plot for the 4.99- $\mu \mathrm{m}$ spheres has basin width of \pm 1.00 at size parameter $x=32.00$ corresponding to a particle diameter of $4.85 \pm 0.15 \mu \mathrm{m}$, consistent with the manufacturer's value.

The data obtained from the 2:1 mixture of spheres are shown in Fig. 7. Although we correct for refraction and fore-

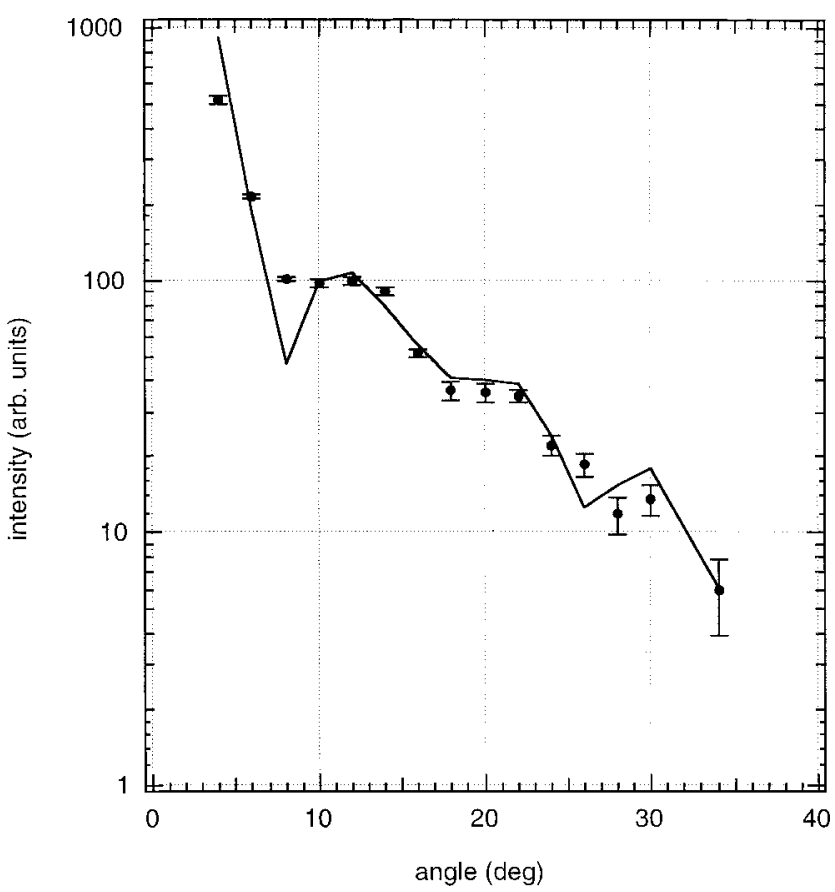

Fig. 7. Light scattered from a suspension of latex spheres made from two sphere sizes. Latex spheres of diameters $4.99 \mu \mathrm{m}(x=32.9)$ and $6.04 \mu \mathrm{m}$ ( $x=39.9$ size parameter) were mixed in a 2:1 ratio. Data and optimally scaled theory curve are shown.

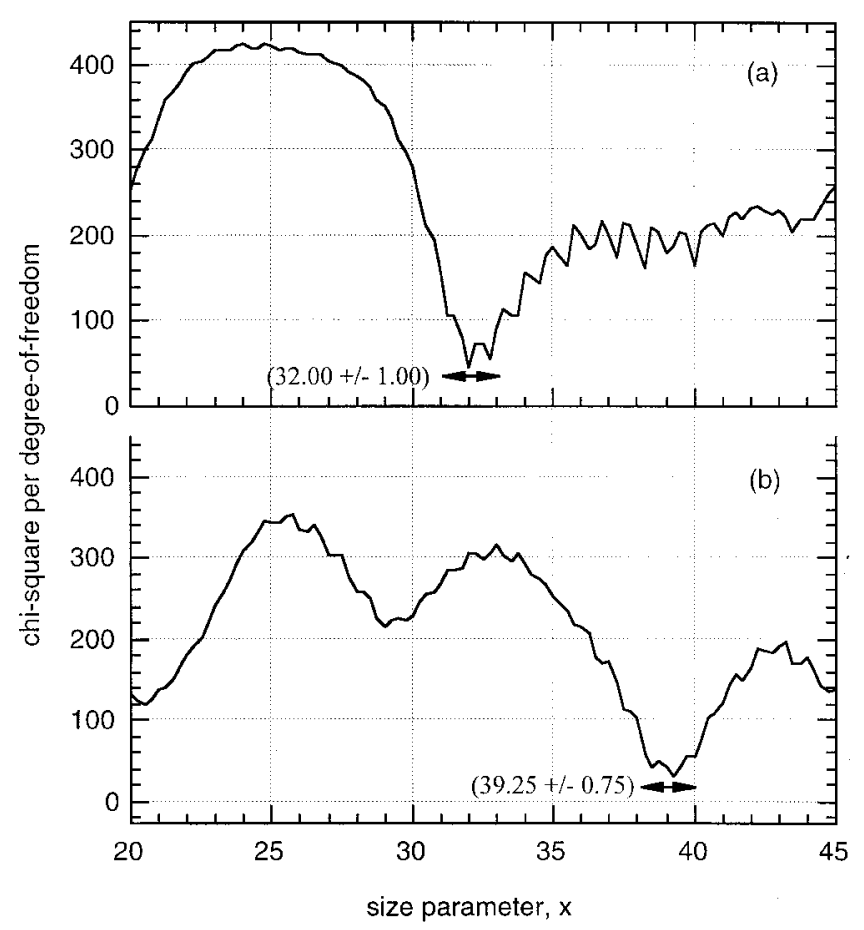

Fig. 8. Chi-square per degree of freedom calculated for the fit as function of size parameter. (a) $4.99-\mu \mathrm{m}$-diam spheres fit to a size parameter of $x$ $=32.00 \pm 1.00$, or $4.85 \pm 0.15 \mu \mathrm{m}$. (b) $6.04-\mu \mathrm{m}$-diam spheres fit to a size parameter of $x=39.25 \pm 0.75$, or $5.95 \pm 0.11 \mu \mathrm{m}$.

shortening effects, we cannot fit the data to the size parameters and relative concentrations using the fitting routine developed. However, we can use the routine to generate corrected theoretical scattering curves that best fit the data, thus allowing an easy comparison of data with theory.

\section{CONCLUSIONS}

We have presented a technique for sizing spherical particles with diameters of the order of $1 \mu \mathrm{m}$ using simple light scattering measurements which are easily implemented in the undergraduate physics lab. Unlike other methods available for particle sizing, our method is cheap, requires only standard laboratory equipment, and is easily implemented. In addition, the numerical methods we have developed and employed are straightforward, highly effective, and can achieve any desired accuracy.

\section{ACKNOWLEDGMENTS}

Acknowledgment is made to the Donors of The Petroleum Research Fund, administered by the American Chemical Society, for partial support of this research, and for support by an award from Research Corporation.

\section{APPENDIX A: MIE SCATTERING COEFFICIENTS}

We derive here the expressions for the scattered electromagnetic field for a plane wave incident upon a homogeneous sphere. For a more detailed derivation, see Bohren and Huffman's book, ${ }^{6}$ upon which we base our derivation. Again, this derivation is mathematically complex; it is included here so that the interested student can find the necessary theory and laboratory discussion in a single document. 
First, we need a convenient way to deal with vector field functions. We begin with the macroscopic Maxwell equations for time-harmonic fields in a given material; that is, for fields of the form

$$
\begin{aligned}
& \mathbf{E}_{i}=\mathbf{E}_{0} \exp (i(\mathbf{k} \cdot \mathbf{x}-\omega t)), \\
& \mathbf{H}_{i}=\mathbf{H}_{0} \exp (i(\mathbf{k} \cdot \mathbf{x}-\omega t)),
\end{aligned}
$$

where $\mathbf{E}$ and $\mathbf{H}$ are the electric and magnetic fields, respectively, $\mathbf{k}$ is the wave vector appropriate for the medium, and $\omega$ is the frequency of the wave.

These fields satisfy the following Maxwell equations:

$$
\begin{aligned}
& \nabla \cdot \mathbf{E}=0, \quad \nabla \cdot \mathbf{H}=0, \\
& \nabla \times \mathbf{E}=i \omega \mu \mathbf{H}, \quad \nabla \times \mathbf{H}=-i \omega \epsilon \mathbf{E}
\end{aligned}
$$

for $\epsilon$ and $\mu$, the electric and magnetic permeability of the material. It is a simple matter to show, using vector identities, that such fields must consequently satisfy the vector wave equations,

$$
\nabla^{2} \mathbf{E}+k^{2} \mathbf{E}=0, \quad \nabla^{2} \mathbf{H}+k^{2} \mathbf{H}=0,
$$

where $k^{2}=\omega^{2} \epsilon \mu$.

To find solutions to the vector wave equation, we construct the following vectors:

$$
\mathbf{M} \equiv \nabla \times(\mathbf{r} \psi), \quad \mathbf{N} \equiv \frac{\nabla \times \mathbf{M}}{k}
$$

where $\mathbf{r}$ is the radius vector in spherical coordinates and $\psi$ is an arbitrary scalar function of position. Again using vector identities, it can be shown that $\mathbf{M}$ and $\mathbf{N}$ satisfy the vector wave equation, and hence Maxwell's equations, if $\psi$ satisfies the scalar wave equation,

$$
\nabla^{2} \psi+k^{2} \psi=0
$$

which, in spherical coordinates, is

$$
\begin{aligned}
& \frac{1}{r^{2}} \frac{\partial}{\partial r}\left(r^{2} \frac{\partial \psi}{\partial r}\right)+\frac{1}{r^{2} \sin \theta} \frac{\partial}{\partial \theta}\left(\sin \theta \frac{\partial \psi}{\partial \theta}\right)+\frac{1}{r^{2} \sin ^{2} \theta} \frac{\partial^{2} \psi}{\partial \phi^{2}} \\
& \quad+k^{2} \psi=0 .
\end{aligned}
$$

Therefore we need only find an expression for the scalar function $\psi$ in order to find vector solutions $\mathbf{M}$ and $\mathbf{N}$.

To solve for $\psi$ we use separation of variables, $\psi(r, \theta, \phi)$ $=R(r) \Theta(\theta) \Phi(\phi)$. This yields separated equations

$$
\begin{aligned}
& \frac{d^{2} \Phi}{d \phi^{2}}+m^{2} \Phi=0, \\
& \frac{1}{\sin \theta} \frac{d}{d \theta}\left(\sin \theta \frac{d \Theta}{d \theta}\right)+\left[n(n+1)-\frac{m^{2}}{\sin ^{2} \theta}\right] \Theta=0, \\
& \frac{d}{d r}\left(r^{2} \frac{d R}{d r}\right)+\left[k^{2} r^{2}-n(n+1)\right] R=0
\end{aligned}
$$

with separation constants $m$ and $n$. Making a change of variable to $\cos \theta$ and $\rho=k r$, we find the following solutions:

$$
\begin{aligned}
& \Phi=\{\cos (m \phi), \sin (m \phi)\}, \\
& \Theta=\left\{P_{n}^{m}(\cos \theta)\right\}, \\
& R=\left\{j_{n}(\rho), y_{n}(\rho)\right\},
\end{aligned}
$$

where $P_{n}^{m}$ is the Legendre function of the first kind of degree $n$, order $m$, and $j_{n}$ and $y_{n}$ are the spherical Bessel functions of order $n$ of the first and second kind, respectively. The requirement of single valuedness for $\psi$ forces $m$ to be an integer, and we get no new functions if $m$ is nonpositive, so we will require $m$ to be a positive integer. The Legendre functions determine the allowed values of $n$ to be $\{m, m$ $+1, \ldots\}$. Thus we have determined a complete set of basis functions that can be used to construct $\psi$, and hence the corresponding vector spherical harmonics,

$$
\begin{aligned}
\mathbf{M}_{e m n}= & \frac{-m}{\sin \theta} \sin (m \phi) P_{n}^{m}(\cos \theta) z_{n}(\rho) \mathbf{e}_{\theta} \\
& -\cos (m \phi) \frac{d P_{n}^{m}(\cos \theta)}{d \theta} z_{n}(\rho) \mathbf{e}_{\phi}, \\
\mathbf{M}_{o m n}= & \frac{m}{\sin \theta} \cos (m \phi) P_{n}^{m}(\cos \theta) z_{n}(\rho) \mathbf{e}_{\theta} \\
& -\sin (m \phi) \frac{d P_{n}^{m}(\cos \theta)}{d \theta} z_{n}(\rho) \mathbf{e}_{\phi}
\end{aligned}
$$

for $\mathbf{M}$ and $\mathbf{N}$,

$$
\begin{aligned}
\mathbf{N}_{e m n}= & \frac{z_{n}(\rho)}{\rho} \cos (m \phi) n(n+1) P_{n}^{m}(\cos \theta) \mathbf{e}_{r} \\
& +\cos (m \phi) \frac{d P_{n}^{m}(\cos \theta)}{d \theta} \frac{1}{\rho} \frac{d}{d \rho}\left[\rho z_{n}(\rho)\right] \mathbf{e}_{\theta} \\
& -m \sin (m \phi) \frac{P_{n}^{m}(\cos \theta)}{\sin \theta} \frac{1}{\rho} \frac{d}{d \rho}\left[\rho z_{n}(\rho)\right] \mathbf{e}_{\phi}, \\
\mathbf{N}_{o m n}= & \frac{z_{n}(\rho)}{\rho} \sin (m \phi) n(n+1) P_{n}^{m}(\cos \theta) \mathbf{e}_{r} \\
& +\sin (m \phi) \frac{d P_{n}^{m}(\cos \theta)}{d \theta} \frac{1}{\rho} \frac{d}{d \rho}\left[\rho z_{n}(\rho)\right] \mathbf{e}_{\theta} \\
& +m \cos (m \phi) \frac{P_{n}^{m}(\cos \theta)}{\sin \theta} \frac{1}{\rho} \frac{d}{d \rho}\left[\rho z_{n}(\rho)\right] \mathbf{e}_{\phi}
\end{aligned}
$$

can be used to construct our solution vectors $\mathbf{M}$ and $\mathbf{N}$, where the subscripts $o$ and $e$ denote using sine and cosine functions (odd and even) for the $\theta$ dependence, and where the $z_{n}$ 's are either of the spherical Bessel functions.

We must now express the incoming plane wave in this basis. In spherical coordinates, an $x$ polarized wave traversing the $z$ direction is given by

$$
\begin{aligned}
& \mathbf{E}_{i}=E_{0} \exp (i k r \cos \theta) \mathbf{e}_{x}, \\
& \mathbf{e}_{x}=\sin \theta \cos \phi \mathbf{e}_{r}+\cos \theta \cos \phi \mathbf{e}_{\theta}-\sin \phi \mathbf{e}_{\phi} .
\end{aligned}
$$

We determine the coefficients of the infinite vector spherical harmonic expansion of the plane wave by using the orthogonality of the basis functions, rejecting harmonics that involve $y_{n}$ since they diverge at the origin. After a bit of tricky integration, we get the following result. We find that $\mathbf{B}_{\text {emn }}$ and $\mathbf{A}_{\text {omn }}$ vanish for all $m$ and $n$, and all coefficients with $m \neq 1$ vanish, producing a considerable simplification:

$$
\mathbf{E}_{i}=E_{0} \sum_{n=1}^{\infty} i^{n} \frac{2 n+1}{n(n+1)}\left(\mathbf{M}_{o 1 n}^{(1)}-i \mathbf{N}_{e 1 n}^{(1)}\right),
$$


where the superscript (1) denotes a radial dependence given by the spherical Bessel function of the first kind. The expression for $\mathbf{H}$ follows from taking the curl of $\mathbf{E}$.

We now solve for the internal and scattered fields, given the incident field above. In order to do this, we must impose boundary conditions on the fields at the surface of the sphere:

$$
\left(\mathbf{E}_{i}+\mathbf{E}_{s}-\mathbf{E}_{1}\right) \times \mathbf{e}_{r}=\left(\mathbf{H}_{i}+\mathbf{H}_{s}-\mathbf{H}_{1}\right) \times \mathbf{e}_{\mathbf{r}}=0,
$$

where $\mathbf{E}_{i}$ is the incident electric field, $\mathbf{E}_{s}$ is the scattered electric field, and $\mathbf{E}_{1}$ is the electric field inside the sphere, with the magnetic field quantities similarly defined. These conditions require the field components tangential to the boundary to be continuous, which is a sufficient condition for conservation of energy.

We expand the internal and scattered fields in our basis, with coefficients to be determined. Inside the sphere, we must again reject the $y_{n}$ 's because we require finiteness at the origin. In addition, the boundary conditions and the form of the incident wave then require that the coefficients of the scattered and internal fields must vanish for $m \neq 1$. So, inside the sphere we have

$$
\begin{aligned}
& \mathbf{E}_{1}=\sum_{n=1}^{\infty} E_{n}\left(c_{n} \mathbf{M}_{o 1 n}^{(1)}-i d_{n} \mathbf{N}_{e 1 n}^{(1)}\right), \\
& \mathbf{H}_{1}=\frac{-k_{1}}{\omega \mu_{1}} \sum_{n=1}^{\infty} E_{n}\left(d_{n} \mathbf{M}_{e 1 n}^{(1)}+i c_{n} \mathbf{N}_{o 1 n}^{(1)}\right),
\end{aligned}
$$

where $E_{n}=E_{0} i^{n} 2 n+1 / n(n+1)$ and $c_{n}$ and $d_{n}$ are coefficients to be determined. Outside the sphere we must use both types of spherical Bessel functions. However, if we switch our basis to spherical Hankel functions defined by

$$
\begin{aligned}
& h_{n}^{(1)}(\rho)=j_{n}(\rho)+i y_{n}(\rho), \\
& h_{n}^{(2)}(\rho)=j_{n}(\rho)-i y_{n}(\rho),
\end{aligned}
$$

we can simplify things. The asymptotic behavior of the Hankel functions for $k r \gg n^{2}$ corresponds to an incoming and outgoing spherical wave:

$$
\begin{aligned}
& h_{n}^{(1)}(k r) \rightarrow \frac{(-i)^{n} \exp (i k r)}{i k r}, \\
& h_{n}^{(2)}(k r) \rightarrow \frac{-i^{n} \exp (-i k r)}{i k r} .
\end{aligned}
$$

We only need the outgoing wave, $h_{n}^{(1)}$, so we can assume the coefficients vanish for the other functions. So, our scattered field is

$$
\begin{aligned}
& \mathbf{E}_{s}=\sum_{n=1}^{\infty} E_{n}\left(i a_{n} \mathbf{N}_{e 1 n}^{(3)}-b_{n} \mathbf{M}_{o 1 n}^{(3)}\right), \\
& \mathbf{H}_{s}=\frac{k}{\omega \mu} \sum_{n=1}^{\infty} E_{n}\left(i b_{n} \mathbf{N}_{o 1 n}^{(3)}+a_{n} \mathbf{M}_{e 1 n}^{(3)}\right),
\end{aligned}
$$

where the superscript (3) indicates radial dependence given by the first (outgoing wave) Hankel function.

Now, the boundary conditions provide us with four independent equations when written in component form. The orthogonality of the basis functions allows us to solve, for each $n$, for the four unknown coefficients $a_{n}, b_{n}, c_{n}$, and $d_{n}$. We get four linear equations,

$$
\begin{aligned}
& j_{n}(m x) c_{n}+h_{n}^{(1)}(x) b_{n}=j_{n}(x), \\
& \mu\left[m x j_{n}(m x)\right]^{\prime} c_{n}+\mu_{1}\left[x h_{n}^{(1)}(x)\right]^{\prime} b_{n}=\mu_{1}\left[x j_{n}(x)\right]^{\prime}, \\
& \mu m j_{n}(m x) d_{n}+\mu_{1} h_{n}^{(1)}(x) a_{n}=\mu_{1} j_{n}(x), \\
& {\left[m x j_{n}(m x)\right]^{\prime} d_{n}+m\left[x h_{n}^{(1)}(x)\right]^{\prime} a_{n}=m\left[x j_{n}(x)\right]^{\prime},}
\end{aligned}
$$

where the prime indicates a derivative, and $x$, the "size parameter,' and $m$, the relative refractive index, are defined by

$$
x=k a=\frac{2 \pi N a}{\lambda}, \quad m=\frac{k_{1}}{k}=\frac{N_{1}}{N} .
$$

These can be solved for the following coefficients:

$$
\begin{aligned}
& a_{n}=\frac{\mu m^{2} j_{n}(m x)\left[x j_{n}(x)\right]^{\prime}-\mu_{1} j_{n}(x)\left[m x j_{n}(m x)\right]^{\prime}}{\mu m^{2} j_{n}(m x)\left[x h_{n}^{(1)}(x)\right]^{\prime}-\mu_{1} h_{n}^{(1)}(x)\left[m x j_{n}(m x)\right]^{\prime}}, \\
& b_{n}=\frac{\mu_{1} j_{n}(m x)\left[x j_{n}(x)\right]^{\prime}-\mu j_{n}(x)\left[m x j_{n}(m x)\right]^{\prime}}{\mu_{1} j_{n}(m x)\left[x h_{n}^{(1)}(x)\right]^{\prime}-\mu h_{n}^{(1)}(x)\left[m x j_{n}(m x)\right]^{\prime}} .
\end{aligned}
$$

Now that we have expressions for the scattered field at any point in space, we can determine the coefficients to any desired accuracy by summing the well-known expressions for Bessel functions to as many terms as necessary. These coefficients can, in turn, be multiplied by the basis functions and summed to any desired degree of accuracy in order to get the scattered field at any point in space. We are interested in the dependence of the field when $r$ is fixed. If we set $\phi=\pi / 2$ and let $\theta$ vary we get the angular dependence of scattered field for incoming light polarized perpendicular to the scattering measurement plane. On the other hand, if we fix $\theta=\pi / 2$ and let $\phi$ vary, we get the angular dependence of the scattered field when the incoming light is polarized parallel to the scattering measurement plane. Note that the form of our separated solution shows that our choice of where to fix $r$ only determines a scale factor for the curves, and therefore is immaterial, since we will be fitting to an arbitrary scaling factor anyway. In fact, there is no need for us to evaluate the radial function whatsoever, or for that matter, the angular function that we hold fixed (either $\phi$ or $\theta$, depending on the polarization); we can simply take them as unity.

\section{APPENDIX B: CORRECTION FOR REFRACTION AND FORESHORTENING}

Here we briefly discuss the techniques employed in correcting for Snell's law and the foreshortening effect. As the laser travels through the cuvette, it illuminates a horizontal column of scatterers. The approach taken here is to discretize this column into a reasonable number (we generally found 20 to be more than sufficient) and correct the problem on a scatterer by scatterer basis. The geometry of the correction for an arbitrary scatterer a distance $x$ from the front face of the cuvette is shown in Fig. 9. Using simple techniques from analytic geometry, one can show that the following equations hold:

$$
\begin{aligned}
& R \cos \phi=\frac{L}{2}+r \cos \theta_{0}, \\
& R \sin \phi=x \tan \theta+r \sin \theta_{0}, \\
& \sin \theta_{0}=N \sin \theta,
\end{aligned}
$$

where the final equation follows directly from Snell's law taking the index of refraction of air to be unity. It is not 


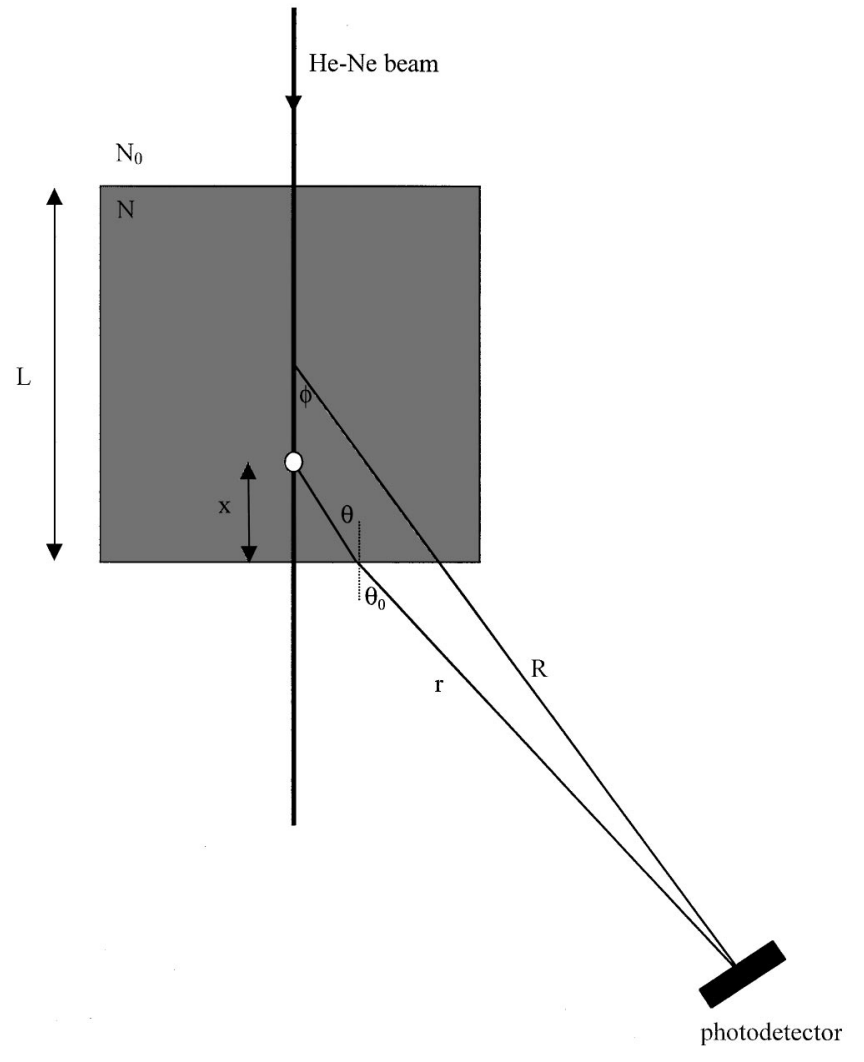

Fig. 9. The geometry of the Snell's law (refraction) and foreshortening corrections. $R$ is the length from the center of the cuvette to the photodetector; $L$ is the length of a cuvette side; $r$ is the distance the refracted light travels from the cuvette face to the photodetector; $N$ is the index of refraction of the fluid (water, in our case) which holds the latex spheres, and $N_{0}$ is the index of refraction of the medium outside of the cuvette (air, in our case).

difficult to solve these equations for a closed-form, albeit inelegant, expression for $\phi$ as a function of $\theta$. However, this is of little use for our correction program, as we need the inverse function; given a detector angle $\phi$ we need to determine what angle $\theta$ a given sphere must scatter at in order to reach our detector. Because a closed-form expression for the inverse function is not forthcoming, we used numerical methods to invert the functions as needed.

In order to get the theoretical corrected scattering curve, we discretized the column of scatterers and treated each one separately. For each scatterer, we integrated numerically the scattered light that reaches the detector over the angular resolution of the detector at the specified angle. We took the angular resolution of our detector to be $1^{\circ}$. It is important to integrate over a finite detector range rather than treat the detector as a point, in order to take into account the significant variations in intensity at various $\phi$ intervals caused by variations with $\phi$ of $d \theta / d \phi$.

\section{APPENDIX C: DESCRIPTION OF THE SOFTWARE DEVELOPED}

We briefly describe the programs used in our analysis. The first program, entitled "MIETABLE,' is the MIEV0 program (originally written in FORTRAN but converted by us to $\mathrm{C}$ using the public domain package F2C). Some minor modifications were made to the code specifically to facilitate the quick generation of tables of angular scattering distributions.

The second program, 'SNELL,', inputs a table of Mie scattering intensities generated by MIETABLE, along with parameters describing the geometry of the experimental setup. It outputs a new table of scattering intensities with Snell's law and foreshortening corrections taken into account.

The final program, "FITMIE,', is the simple fitting routine discussed in the main text. It inputs the experimental data (in a simple tab-delimited text format) and the corrected theoretical table generated by SNELL. It returns the best fit size parameter $x$ and the scaling parameter associated with it. There is also an option to output a plot of chi-square as a function of size parameter.

\footnotetext{
${ }^{1}$ Norman J. Dovichi and Fahimeh Zarrin, "Laser Doppler Velocimetry for Submicrometer Particle Size Determination," AIP Conf. Proc. 160 (1), 529-531 (1987).

${ }^{2}$ Jianhong Wang and F. Ross Hallett, "Spherical Particle Size Determination by Analytical Inversion of the UV-Visible-NIR Extinction Spectrum," Appl. Opt. 35 (1), 193-197 (1996).

${ }^{3}$ Mao Ye, Shimin Wang, Yong Lu, Tao Hu, Zhen Zhu, and Yiqian Xu,

"Inversion of Particle-size Distribution from Angular Light Scattering Data with Genetic Algorithms," Appl. Opt. 38 (12), 2677-2685 (1999).

${ }^{4}$ R. M. Drake and J. E. Gordon, “Mie Scattering," Am. J. Phys. 53 (10), 955-962 (1985).

${ }^{5}$ H. C. van de Hulst, Light Scattering by Small Particles (Dover, New York, 1981).

${ }^{6}$ Craig F. Bohren and Donald R. Huffman, Absorption and Scattering of Light by Small Particles (Wiley, New York, 1983).

${ }^{7}$ Warren Wiscombe, "Improved Mie Scattering Algorithms," Appl. Opt. 19 (9), 1505-1509 (1980). Dr. Warren Wiscombe's MIEVo program, written in FORTRAN 77, is available by anonymous ftp from climate.gsfc.nasa.gov in subdirectory pub/wiscombe.
}

\section{LECTURES AND XEROX MACHINES}

These are great for arousing the emotions. As a means of instruction, they ought to have become obsolete when the printing press was invented. We had a second chance when the Xerox machine was invented, but we seem to have muffed it. If you have to lecture, you can at least hand out copies of what you said (or wish you had said).

Ralph P. Boas, Jr., 'Can We Make Mathematics Intelligible?,' in Lion Hunting \& Other Mathematical Pursuits, edited by Gerald L. Alexanderson and Dale H. Mugler (Mathematical Association of America, Washington, 1995 ), p. 236. 